\title{
Odd Khovanov homology
}

\author{
PETER S OZSVÁTH \\ JACOB RASMUSSEN \\ ZOLTÁN SZABÓ
}

\begin{abstract}
We describe an invariant of links in $S^{3}$ which is closely related to Khovanov's Jones polynomial homology. Our construction replaces the symmetric algebra appearing in Khovanov's definition with an exterior algebra. The two invariants have the same reduction modulo 2 , but differ over $\mathbb{Q}$. There is a reduced version which is a link invariant whose graded Euler characteristic is the normalized Jones polynomial.
\end{abstract}

57M25; 57R58

\section{Introduction}

In his influential paper, Khovanov [4] describes a link invariant which associates to a link a bigraded Abelian group whose graded Euler characteristic is the Jones polynomial. His invariant is obtained from a TQFT which associates to a collection of embedded, planar circles the symmetric algebra of the vector space generated by the circles.

Our goal here is to describe a modified version of Khovanov homology - which we call odd Khovanov homology - which associates to a collection of embedded, planar circles the exterior algebra of the vector space generated by the circles. The constructions described here are clearly quite closely related to Khovanov's.

Before giving the construction, we give some motivation. In [12], the first and third authors show that there is a spectral sequence whose $E_{2}$-term is the Khovanov homology of a link $L \subset S^{3}$, with coefficients taken in $\mathbb{Z} / 2 \mathbb{Z}$, and which converges to the Heegaard Floer homology $\widehat{\mathrm{HF}}$ of the branched double-cover of $L$. Our motivation for finding odd Khovanov homology came from our attempts to lift this to a result over $\mathbb{Z}$. Consequently, it is natural to make the following:

Conjecture 1.1 Let $L \subset S^{3}$ be a link. There is a spectral sequence whose $E_{2}$-term is the reduced odd Khovanov homology of $L$ and whose $E_{\infty}$-term is the Heegaard Floer homology of the branched double-cover of $L$ (with coefficients in $\mathbb{Z}$ ). 
A similar result should hold, with a suitable construction from Seiberg-Witten monopole Floer homology (see Kronheimer and Mrowka [8]) replacing the Heegaard Floer homology of the branched double-cover.

Key features of odd Khovanov homology can be summarized as follows:

- The mod two reductions of the original Khovanov homology (which we will refer to presently as the even construction) and odd Khovanov homology coincide (see Proposition 1.7 below).

- Like the even theory, odd Khovanov homology categorifies the unnormalized Jones polynomial (see Proposition 1.5 below).

- The odd theory has a reduced version, which is a link invariant categorifying the usual Jones polynomial (see Proposition 1.8 below). Note that the even theory also has a reduced version (see Khovanov [5]), but this depends on a choice of a link component.

Since the first appearance of this preprint, there have been several further developments related to the constructions presented in this paper; see for example Bloom [2], Putyra [13], Shumakovitch [16] and the third author [17].

\subsection{A projective TQFT}

Consider the category $\mathfrak{C}$ of compact one-manifolds and compact, orientable cobordisms between them. The starting point for the construction of the odd Khovanov homology is a "projective functor" $F$ from $\mathfrak{C}$ to the category of graded $\mathbb{Z}$-modules. This functor is "projective" in the sense that the map assigned to a morphism is well-defined only up to an overall sign.

An object $S \in \mathrm{Ob}(\mathfrak{C})$ is a disjoint union of circles. Given such an $S$, we let $V(S)$ denote the free abelian group generated by its components, and define $F(S)=\Lambda^{*} V(S)$. Morphisms in $\mathfrak{C}$ are generated by four types of elementary morphism: zero-handle additions (or births), one-handle additions where the feet of the one handle lie on two different components (merges), one-handle additions where the feet lie on the same component (splits), and two-handle additions (deaths). We will be most interested in the morphisms corresponding to one-handle additions.

Suppose that $M: S_{1} \rightarrow S_{2}$ is a merge cobordism which joins the circles $a_{1}$ and $a_{2}$ in $S_{1}$. Then there is a natural identification $V\left(S_{2}\right) \cong V\left(S_{1}\right) /\left(a_{1}-a_{2}\right)$, and we define

$$
F_{M}: \Lambda^{*} V\left(S_{1}\right) \longrightarrow \Lambda^{*} V\left(S_{2}\right)
$$

to be the map induced by the projection $V\left(S_{1}\right) \rightarrow V\left(S_{1}\right) /\left(a_{1}-a_{2}\right) \cong V\left(S_{2}\right)$. 
Next, suppose that $M: S_{1} \rightarrow S_{2}$ is a split cobordism in which a single circle $a$ in $S^{1}$ divides to form two circles $a_{1}$ and $a_{2}$ in $S_{2}$. Then the natural identification $V\left(S_{1}\right) \cong V\left(S_{2}\right) /\left(a_{1}-a_{2}\right)$ induces an identification

$$
\Lambda^{*} V\left(S_{1}\right) \cong \Lambda^{*}\left(\frac{V\left(S_{2}\right)}{\left(a_{1}-a_{2}\right)}\right) \cong\left(a_{1}-a_{2}\right) \wedge \Lambda^{*} V\left(S_{2}\right)
$$

The second isomorphism is well-defined only up to sign. We define $F_{M}: \Lambda^{*} V\left(S_{1}\right) \rightarrow$ $\Lambda^{*} V\left(S_{2}\right)$ to be the composition

$$
\Lambda^{*}\left(V\left(S_{1}\right)\right) \stackrel{\cong}{\longrightarrow} \Lambda^{*}\left(\frac{V\left(S_{2}\right)}{\left(a_{1}-a_{2}\right)}\right) \stackrel{\cong}{\longrightarrow}\left(a_{1}-a_{2}\right) \wedge \Lambda^{*} V\left(S_{2}\right) \stackrel{\subset}{\longrightarrow} \Lambda^{*} V\left(S_{2}\right) .
$$

This map sends $1 \in \Lambda^{*} V\left(S_{1}\right)$ to $\pm\left(a_{1}-a_{2}\right) \in \Lambda^{*} V\left(S_{2}\right)$.

For completeness, we record the maps induced by the birth and death cobordisms as well. A birth cobordism $M: S_{1} \rightarrow S_{2}$ induces an inclusion $V\left(S_{1}\right) \rightarrow V\left(S_{2}\right)$, and $F_{M}$ is the induced map. In a death cobordism $M: S_{1} \rightarrow S_{2}$, there is a distinguished component $A$ in $S_{2}$ which is capped off by the two-handle; the map $F_{M}$ is given by contraction with the dual of $a$.

The reader can easily verify that up to sign, these maps satisfy all the identities associated with a TQFT. We could eliminate the sign ambiguity by tensoring with $\mathbb{Z} / 2$; the resulting TQFT can be identified with the $\mathbb{Z} / 2$-reduction of the TQFT used by Khovanov in [4].

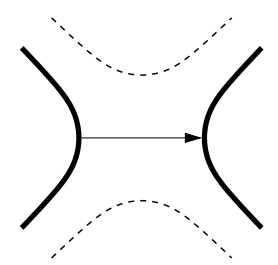

Figure 1: This illustrates an elementary saddle move. The two heavy solid arcs represent the initial union of circles, the dotted ones represent the result of the saddle move, and the oriented arc represents an orientation on the one-handle of the cobordism.

Another approach is to try to deal with the sign ambiguity by decorating our cobordisms. Suppose we have a cobordism $M: S_{1} \rightarrow S_{2}$ with a fixed decomposition into onehandles. Such a cobordism is specified by an $n$-tuple of embedded zero-spheres $Z_{1}, \ldots, Z_{n} \subset S_{1}$. These can be represented diagrammatically by joining the two points of $Z_{i}$ by an arc representing the core of the one-handle and fixing an (arbitrary) orientation on it, as shown in Figure 1. If the cobordism associated to the handle 
addition $Z_{i}$ is a split, we fix the sign of $F_{Z_{i}}$ by requiring that it take 1 to $a_{1}-a_{2}$, where the arrow points from $a_{1}$ to $a_{2}$.

Given two one-handles $\left\{Z_{1}, Z_{2}\right\}$, the induced maps commute up to sign:

$$
F_{Z_{2}} \circ F_{Z_{1}}=\epsilon \cdot F_{Z_{1}} \circ F_{Z_{2}}
$$

When the composite map is nontrivial, the sign of $\epsilon$ can be determined from the combinatorics of how the two $\operatorname{arcs} Z_{1}$ and $Z_{2}$ interact with each other, as illustrated in Figure 2. When $\epsilon=-1$, we call the pair of type $A$; when $\epsilon=+1$, we call the pair of type $C$. There are two remaining cases where the double-composites are trivial. We label these cases by $X$ and $Y$, as shown in Figure 2.

Figure 2 is not meant to be an exhaustive list of all the pictures embedded in $S^{2}$ of all the squares of type $A$ and $C$. For the cases where there is more than one circle, other pictures can be obtained by nesting various circles inside each other. However, we have chosen a representative choice for each of these possible nestings.

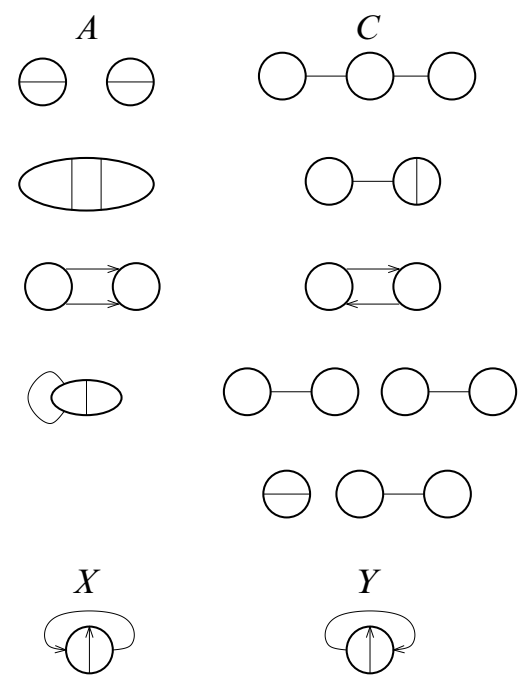

Figure 2: Commutation chart. The set of pairs of arcs can be placed into four categories, types $A, C, X$ and $Y$. In type $A$, the double-composite maps anticommute. In type $C$, they commute. The two remaining cases are labeled $X$ and $Y$ as above. The thicker curves denote components of $S_{1}$, while the thinner arcs represent the one-handles specified by $Z$. Orientations of these one-handles are specified by arrows when they are needed; when they are dropped, it is because the corresponding picture has the stated type for either choice of orientation. 


\subsection{The hypercube of resolutions}

As in the usual Khovanov homology, we start with an oriented link diagram (this will be relevant for calibrating the gradings: ie in the numbers $n_{+}$and $n_{-}$below). In addition to this, in order to define signs in the odd theory, we have to fix some further data, which we call a crossing orientation. (Note that this latter choice is a bookkeeping device, and it will turn out that odd Khovanov homology is independent of this choice, see Lemmas 2.2 and 2.3 below.)

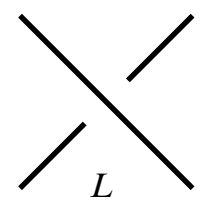

$L$

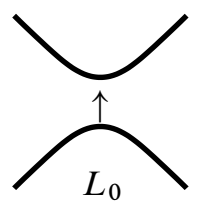

$L_{0}$

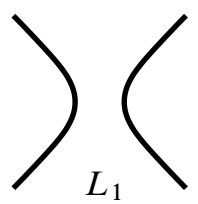

$L_{1}$

Figure 3: Oriented skein moves. The crossing on the left has two resolutions, as illustrated. The choice of crossing orientation is indicated by an arrow in the 0 -resolution.

If $\mathcal{D}$ is a planar diagram of a link, we can define its associated hypercube of oriented resolutions. Specifically, each crossing in the projection has two resolutions $L_{0}$ and $L_{1}$. (These are the resolution conventions of Khovanov's [4]; they are opposite to those from the first and third author's [12].) The crossing can be thought of as giving a cobordism from $L_{0}$ to $L_{1}$ consisting of a single one-handle. A crossing orientation is indicated by a choice at each crossing in the diagram: namely, we fix an orientation on each one-handle. Equivalently, we draw an arrow joining the two segments of the 0 -resolution at each crossing, as illustrated in Figure 3. Let $\mathcal{X}$ denote the set of crossings of the diagram $\mathcal{D}$. Then for each map $I: \mathcal{X} \rightarrow\{0,1\}$, we have an associated embedded one-manifold in the plane, $\mathcal{D}(I)$, which replaces a given crossing $x \in \mathcal{X}$ by its oriented resolution of type $I(x)$.

Given two vertices $I_{0}, I_{1}: \mathcal{X} \rightarrow\{0,1\}$ in the hypercube, we say that there is an oriented edge from $I_{0}$ to $I_{1}$ if there is some $x \in \mathcal{X}$ with the property that

$$
\begin{array}{ll}
I_{0}(x)=0, & I_{1}(x)=1 \\
I_{0}(y)=I_{1}(y) & \text { if } x \neq y .
\end{array}
$$

We let $\mathcal{E}(\mathcal{D})$ and $\mathcal{V}(D)$ denote the set of edges and vertices, respectively. Each vertex $I$ corresponds to an object $\mathcal{D}(I)$ in $\mathfrak{C}$, and each edge in $\mathcal{E}$ corresponds to a morphism. Given $e \in \mathcal{E}$ from $I_{0}$ to $I_{1}$, we let $M_{e}$ denote the corresponding morphism from $\mathcal{D}\left(I_{0}\right)$ to $\mathcal{D}\left(I_{1}\right)$. 
Two-dimensional faces, or squares, in the hypercube of oriented resolutions correspond to pairs of resolutions $I_{00}, I_{11}: \mathcal{X} \rightarrow\{0,1\}$ for which there are exactly two $x_{1}, x_{2} \in \mathcal{X}$ with

$$
\begin{aligned}
I_{00}\left(x_{1}\right) & =0, & & I_{11}\left(x_{1}\right)=1, \\
I_{00}\left(x_{2}\right) & =0, & & I_{11}\left(x_{2}\right)=1, \\
I_{00}(y) & =I_{11}(y) & & \text { if } y \notin\left\{x, x^{\prime}\right\} .
\end{aligned}
$$

Each square face may be classified as one of types $A, C, X$ or $Y$, according to the classification scheme in Figure 2.

Definition 1.2 An edge assignment for a diagram $\mathcal{D}$ is a map $\epsilon: \mathcal{E}(\mathcal{D}) \rightarrow\{ \pm 1\}$. Given an edge assignment $\epsilon$, we say that a square face is even or odd, depending on whether it contains an even or odd number of edges with $\epsilon(e)=-1$. A type $X$ edge assignment is an edge assignment with the property that all faces of type $A$ and $X$ are even and all faces of type $C$ and $Y$ are odd. Similarly, a type $Y$ edge assignment is an edge assignment for which faces of type $A$ and $Y$ are even and faces of type $C$ and $X$ are odd.

The following lemma will be established in Section 2.

Lemma 1.3 Any diagram $\mathcal{D}$ has an edge assignment of type $X$, and one of type $Y$.

\subsection{The chain complex}

Consider the vector space

$$
C(\mathcal{D})=\bigoplus_{I \in \mathcal{V}(\mathcal{D})} \Lambda^{*} V(\mathcal{D}(I))
$$

Given a type $X$ or a type $Y$ edge assignment $\epsilon: \mathcal{E}(\mathcal{D}) \rightarrow\{ \pm 1\}$, we can define an endomorphism $\partial_{\epsilon}$ of $C(\mathcal{D})$ by the formula

$$
\partial_{\epsilon}(v)=\sum_{e, J \in \mathcal{S}} \epsilon(e) \cdot F_{e}(v)
$$

for $v \in \Lambda^{*} V(\mathcal{D}(I))$, where $\mathcal{S}=\{e \in \mathcal{E}(\mathcal{D}), J \in \mathcal{V}(D) \mid e$ goes from $I$ to $J\}$.

We claim that $\partial_{\epsilon}^{2}=0$. Indeed, this relation is satisfied provided

$$
\epsilon\left(e_{1}\right) \epsilon\left(e_{2}\right) F_{e_{1}} \circ F_{e_{2}}+\epsilon\left(e_{3}\right) \epsilon\left(e_{4}\right) F_{e_{3}} \circ F_{e_{4}}=0
$$


whenever we have four edges $\left\{e_{1}, \ldots, e_{4}\right\}$ bounding a square (so that $e_{1} e_{2}$ and $e_{3} e_{4}$ are the two paths from the initial point to the final point). For a square of type $A$ or $C$, this holds by Equation (1), while for squares of type $X$ or $Y$, the relation holds since

$$
F_{e_{1}} \circ F_{e_{2}}=F_{e_{3}} \circ F_{e_{4}}=0 .
$$

Thus the pair $\left(C(\mathcal{D}), \partial_{\epsilon}\right)$ defines a chain complex.

$C(\mathcal{D})$ can be equipped with a bigrading $C_{a, b}(\mathcal{D})$, following [4]. Specifically, we endow the exterior algebra $\Lambda^{*} V(\mathcal{D}(I))$ with the $q$-grading $Q_{0}$ for which $\Lambda^{r} V(\mathcal{D}(I))$ has $q$-grading equal to $\operatorname{dim} V(\mathcal{D}(I))-2 r$. Similarly, we define the initial homological grading $M_{0}$ on $C(\mathcal{D})$ so that $C(\mathcal{D}(I))$ is supported in grading $\sum_{c \in \mathcal{X}} I(c)$. The $q$-grading on $C(\mathcal{D})$ is then given by $Q=Q_{0}+n_{+}-2 n_{-}+M_{0}$, where here $n_{-}$ denotes the number of negative crossings in the diagram, and the homological grading $M=M_{0}-n_{-}$. We write

$$
C(\mathcal{D})=\bigoplus_{m, s \in \mathbb{Z}} C_{m, s}(\mathcal{D})
$$

where $m$ corresponds to the homological grading and $s$ the $q$-grading. Since the differential preserves $Q$-grading and drops $M$-grading by one, the two gradings descend to homology, and we can write

$$
H(\mathcal{D})=\bigoplus_{m, s \in \mathbb{Z}} H_{m, s}(\mathcal{D})
$$

Theorem 1.4 Let $L$ be a link. Fix an oriented projection $\mathcal{D}$ of $L$ and an edge assignment $\epsilon$ of type $X$ or $Y$. The bigraded homology groups of $\left(C(\mathcal{D}), \partial_{\epsilon}\right)$ are independent of the choice of $\mathcal{D}$ and $\epsilon$.

We call the above bigraded homology groups the odd Khovanov homology of the link $L, \mathrm{Kh}^{\prime}(L)$, to distinguish it from ordinary $\operatorname{sl}(2)$ Khovanov homology $\mathrm{Kh}(L)$ (where the variables are "even"). We collect here some properties of $\mathrm{Kh}^{\prime}(L)$ which follow quickly from its construction; proofs will be supplied in Section 4.

Recall that the unnormalized Jones polynomial is characterized by the properties that:

$$
\begin{aligned}
\widehat{J}(\varnothing) & =1, \\
\widehat{J}((\text { unknot }) \cup L) & =\left(q+q^{-1}\right) \cdot \widehat{J}(L), \\
\widehat{J}(\rtimes) & \doteq \widehat{J}(\asymp)-q \cdot \widehat{J}()(),
\end{aligned}
$$

where for $f, g \in \mathbb{Z}\left[q, q^{-1}\right]$, we write $f \doteq g$ if $f= \pm q^{j} \cdot g$ for some $j \in \mathbb{Z}$. 
Proposition 1.5 $\mathrm{Kh}^{\prime}(L)$ categorifies the unnormalized Jones polynomial, in the sense that

$$
\widehat{J}(L)=\sum_{m, s \in \mathbb{Z}}(-1)^{m} \operatorname{rank}\left(\mathrm{Kh}_{m, s}^{\prime}(L)\right) \cdot q^{s}
$$

Exactly as in Khovanov's original construction, the skein relation characterizing the Jones polynomial is replaced by a skein exact sequence; $\mathrm{cf}[4]$ :

Proposition 1.6 There is a long sequence

$$
\longrightarrow \mathrm{Kh}^{\prime}(\asymp) \stackrel{i_{*}}{\longrightarrow} \mathrm{Kh}^{\prime}\left(\text { メ) } \stackrel{\pi_{*}}{\longrightarrow} \mathrm{Kh}^{\prime}()() \stackrel{\partial}{\longrightarrow} \mathrm{Kh}^{\prime}(\asymp) \longrightarrow .\right.
$$

The maps $i_{*}, \pi_{*}$ and $\delta$ are all homogenous with respect to the bigrading; for a precise statement of the grading shifts, see eg Manolescu and the first author's [10] or the second author's [14].

Proposition 1.7 The mod two reduction of $\operatorname{Kh}^{\prime}(L)$ agrees with the mod two reduction of Khovanov's sl(2) homology $\mathrm{Kh}^{\prime}(L)$; ie

$$
\begin{aligned}
& \operatorname{Tor}\left(\mathrm{Kh}_{m+1, s}(L), \mathbb{Z} / 2 \mathbb{Z}\right) \oplus\left(\mathrm{Kh}_{m, s}(L) \otimes \mathbb{Z} / 2 \mathbb{Z}\right) \\
& \quad \text { Tor }\left(\mathrm{Kh}_{m+1, s}^{\prime}(L), \mathbb{Z} / 2 \mathbb{Z}\right) \oplus\left(\mathrm{Kh}_{m, s}^{\prime}(L) \otimes \mathbb{Z} / 2 \mathbb{Z}\right) .
\end{aligned}
$$

Despite their formal similarities, $\mathrm{Kh}^{\prime}$ and $\mathrm{Kh}$ are actually very different groups. The first indication of this fact is given by

Proposition 1.8 There is a bigraded Abelian group $\overline{\mathrm{Kh}}^{\prime}(L)$ with the property that

$$
\mathrm{Kh}_{m, s}^{\prime}(L) \cong \overline{\mathrm{Kh}}_{m, s-1}^{\prime} \oplus \overline{\mathrm{Kh}}_{m, s+1}^{\prime}(L) .
$$

We call $\overline{\mathrm{Kh}}^{\prime}(L)$ the reduced odd Khovanov homology. The bigraded group $\overline{\mathrm{Kh}}^{\prime}(L)$ categorifies the ordinary Jones polynomial $J(L)$, which is defined by the relation $\left(q+q^{-1}\right) \cdot J(L)=\widehat{J}(L)$. It is analogous to the reduced Khovanov homology $\overline{\mathrm{Kh}}$ defined in Khovanov's [5], which also categorifies the ordinary Jones polynomial, but there are some differences. In the definition of the ordinary reduced Khovanov homology, one fixes a component of $L$; different choices of component can lead to different answers (which can be seen, for example, by considering the disjoint union of the trefoil and an unknot). By contrast, $\overline{\mathrm{Kh}}^{\prime}$ is a link invariant. Moreover, the relation between $\overline{\mathrm{Kh}}^{\prime}$ and $\mathrm{Kh}^{\prime}$ is simpler than the relation between $\overline{\mathrm{Kh}}$ and $\mathrm{Kh}$.

For small knots $K$, the groups $\overline{\mathrm{Kh}}^{\prime}(K)$ and $\overline{\mathrm{Kh}}(K)$ are isomorphic. Indeed, we have: 
Proposition 1.9 If $L$ is a nonsplit alternating link, then $\overline{\mathrm{Kh}}^{\prime}(L) \cong \overline{\mathrm{Kh}}(L)$.

However, there are many nonalternating knots for which the two groups are not isomorphic. ${ }^{1}$

This paper is organized as follows. In Section 2 we construct the edge assignments needed to construct the chain complex (Lemma 1.3), and show that the isomorphism class of the complex is independent of the choice of sign assignment and the orientations. In Section 3, we show that odd Khovanov homology is independent of the link projection. The arguments here follow closely the invariance proof of Khovanov's $s l(2)$ theory (see Khovanov [4], and also Bar-Natan [1] and Khovanov and Rozansky [6; 7]). In Section 4, we establish the basic properties of this construction enumerated above. Finally, in Section 5, we exhibit some calculations of these groups.

Acknowledgements We wish to thank Mikhail Khovanov, Tomasz Mrowka, and Paul Seidel for their encouragement during the preparation of this manuscript.

P S Ozsváth was supported by NSF grants number DMS-0505811 and FRG-0244663. J Rasmussen was supported by NSF grant number DMS-0603940 and a Sloan Fellowship. Z Szabó was supported by NSF grants number DMS-0704053 and DMS-1006006.

\section{Existence and uniqueness of edge assignments}

Our first goal in this section is to prove Lemma 1.3, which allows us to construct the chain complex for odd Khovanov homology. We then make some preliminary steps towards the proof of Theorem 1.4 by showing that the isomorphism type of the complex $\left(C(\mathcal{D}), \partial_{\epsilon}\right)$ does not depend on $\epsilon$ or on the choice of orientation at the crossings.

Lemma 1.3 will follow quickly from the following lemma about cubes in the hypercube of oriented resolutions; but before stating this lemma, we note that a cube in the hypercube of resolutions is determined by a pair of resolutions

$$
I_{000}, I_{111}: \mathcal{X} \longrightarrow\{0,1\}
$$

with the property that there are three crossings $x_{1}, x_{2}, x_{3} \in \mathcal{X}$ such that

$$
\begin{array}{ll}
I_{000}\left(x_{i}\right)=0, \quad I_{111}\left(x_{i}\right)=1 & \text { for } i=1,2,3, \\
I_{000}(y)=I_{111}(y) & \text { if } y \notin\left\{x_{1}, x_{2}, x_{3}\right\} .
\end{array}
$$

\footnotetext{
${ }^{1}$ Manturov [11] formulates a version of Khovanov homology for virtual knots using exterior algebras as well; however, his constructions are different from the ones here. Indeed, for classical knots, his groups agree with Khovanov's.
} 
Lemma 2.1 Each cube in the hypercube of resolutions contains an even number of squares of type $A$ and $X$. Similarly, each cube contains an even number of squares of type $A$ and $Y$.

Proof This is a case-by-case analysis according to the different possible combinatorial types of cubes in the hypercube of resolutions. Specifically, a cube corresponds to eight resolutions, which are given a partial ordering, with a unique minimal element. The cube is determined by this minimal element $\left(I_{000}\right)$ and the three oriented arcs connecting various components (corresponding to three crossings in the original projection). We disregard all the unknotted circles which do not meet these three arcs. This leaves us between one and four circles, which are connected by the oriented arcs (in the plane). We enumerate the possible connected diagrams in Figure 4.
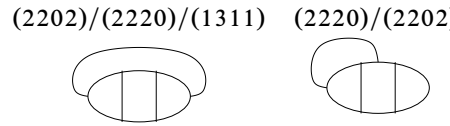

$(6000) /(4200)$
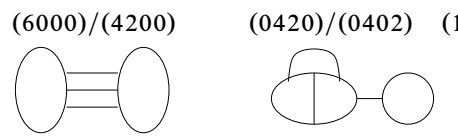

$(1311) /(0420) /(0402)$

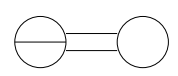

$(2400) /(0600)$

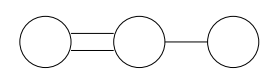

(6000)

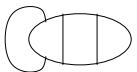

$(2400) /(4200)$
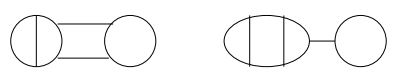

(2400)

(2400)

$(6000)$
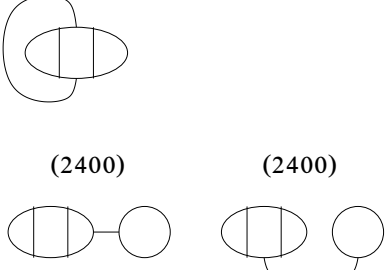

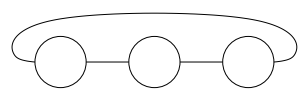

(0600)

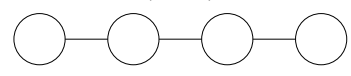

Figure 4: Different types of cubes in the hypercube of resolutions. Each cube is labeled with choices of distributions of faces of type $A, C, X$ and $Y$. The different possibilities correspond to various orientations on the arcs connecting them.

Let $a, c, x$ and $y$ denote the number of squares of types $A, C, X$ and $Y$ respectively in each cube. The lemma states that both $a+x$ and $a+y$ are even. This is an easy verification. Note that the six squares in each cube are realized by choosing one of the three arcs, and either dropping it, or performing surgery along it. In Figure 5, we have pictured the possibilities for the second type of cube appearing in Figure 4, with one of the eight different possible choices of orientations. We see that there are two 
squares of type $C$, two of type $A$ and two of type $Y$. The number of squares of types $C, A, X$ and $Y$ in the other cases is indicated in Figure 4. We leave it to the reader to verify that in all cases, $a+x$ and $a+y$ are even. This is a straightforward if tedious computation. In some cases, it is helpful to note that for most squares, the $A$ or $C$ type is independent of the choice of orientations of the arcs. For example, in the last picture in Figure 4 (four distinct circles connected by three arcs), all the squares are of type $C$ regardless of the orientations of the arcs.

If the diagram is disconnected, it has a component with only one arc. We orient the cube so that the four edges corresponding to this arc are vertical; then the top and bottom faces are of the same type. If the vertical edges correspond to merges, all four vertical faces are of type $C$. If they are splits, each merge in the top face corresponds to a vertical face of type $C$, and each split corresponds to a vertical face of type $A$. The number of merges and splits in the top face are both even, so the claim holds in this case as well.
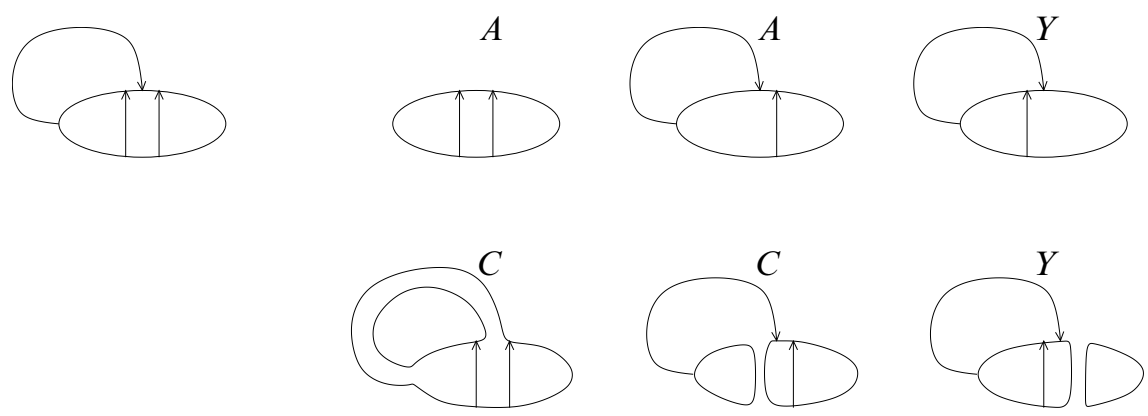

Figure 5: Counting types of squares in a cube. Consider the cube corresponding to the diagram on the left-hand side of the figure. The six square faces of the cube are gotten by either forgetting or performing surgery along one of the arcs. These are shown and classified on the right.

Proof of Lemma 1.3 Let $G=\mathbb{Z}^{*} \cong \mathbb{Z} / 2$ be the multiplicative group with elements \pm 1 . We consider the hypercube $Q$ of oriented resolutions as a cell complex. We can define on it a 2 -cochain $\phi \in C^{2}(Q ; G)$ which associates to each face of type $A$ or $X$ the element $1 \in G$, and to each face of type $C$ or $Y$ the number $-1 \in G$. Lemma 2.1 shows that $\phi$ is a cocycle. Since the cube is contractible, $\phi$ must be a coboundary. Concretely, this means that there is some function $\epsilon: \mathcal{E} \rightarrow\{ \pm 1\}$ with the property that $\epsilon\left(e_{1}\right) \epsilon\left(e_{2}\right) \epsilon\left(e_{3}\right) \epsilon\left(e_{4}\right)=\phi(\sigma)$, where $e_{1}, \ldots, e_{4}$ are the four edges of the square $\sigma$. This is the required edge assignment of type $X$. The same remarks hold for constructing an edge assignment of type $Y$. 
Lemma 2.2 If $\epsilon$ and $\epsilon^{\prime}$ are two edge assignments of the same type ( $X$ or $Y$ ), then the chain complex $\left(C(\mathcal{D}), \partial_{\epsilon}\right)$ is isomorphic to $\left(C(\mathcal{D}), \partial_{\epsilon^{\prime}}\right)$.

Proof Suppose that $\epsilon$ and $\epsilon^{\prime}$ are two edge assignments of the same type. Then $\epsilon \cdot \epsilon^{\prime}$ is a one-dimensional cocycle, so it can be realized as the coboundary of a zero-cochain; ie we have a map $\eta: \mathcal{V}(\mathcal{D}) \rightarrow\{ \pm 1\}$ with $\eta\left(v_{1}\right) \eta\left(v_{2}\right)=\epsilon(e) \epsilon^{\prime}(e)$ if $v_{1}$ and $v_{2}$ are the endpoints of $e$. Consider the endomorphism $\Phi: C(\mathcal{D}) \rightarrow C(\mathcal{D})$ which, when restricted to $C(\mathcal{D}(I))$, is given by multiplication by $\eta(I)$. It is straightforward to verify that $\Phi$ is an isomorphism of chain complexes, from $\left(C(\mathcal{D}), \partial_{\epsilon}\right)$ to $\left(C(\mathcal{D}), \partial_{\epsilon^{\prime}}\right)$.

Lemma 2.3 If $\mathcal{D}$ and $\mathcal{D}^{\prime}$ are two choices of crossing-orientations with same underlying diagram, then there are edge assignments of the same type $\epsilon$ and $\epsilon^{\prime}$ with $\left(C(\mathcal{D}), \partial_{\epsilon}\right) \cong\left(C\left(\mathcal{D}^{\prime}\right), \partial_{\epsilon^{\prime}}\right)$.

Proof It suffices to consider the case where we change the orientation at a single crossing $c$. If we denote the maps in the new cube of resolutions by $F_{e}^{\prime}$, then we can write $F_{e}^{\prime}=\alpha(e) \cdot F_{e}$. Here $\alpha(e)=-1$ if the crossing associated to $e$ is $c$ and the corresponding cobordism is a split, and $\alpha(e)=1$ otherwise. If $\phi^{\prime}$ is the class in $C^{2}(Q ; G)$ associated to the new cube, we claim that $\phi^{\prime}=\phi \cdot d \alpha$. For faces of type $A$ and $C$, this is obvious, while for a face $\sigma$ of type $X$ or $Y$, reversing the orientation of one of the two arcs in $\sigma$ switches types $X$ and $Y$, so $\phi^{\prime}(\sigma)=-\phi(\sigma)$. On the other hand, two of the edges in this face correspond to one-handle attachments near $c$. Exactly one of them is a split (and thus has $\alpha(e)=-1$ ). It follows that if $\epsilon$ is an edge assignment of type $X$ for the old cube, $\alpha \cdot \epsilon$ is an edge assignment of type $X$ for the new cube, and the boundary map $\partial_{\alpha \cdot \epsilon}$ is in the new complex is exactly the same as $\partial_{\epsilon}$ in the old one.

Lemma 2.4 If $\epsilon$ and $\epsilon^{\prime}$ are sign assignments of opposite types, then there is an isomorphism $\left(C, \partial_{\epsilon}\right) \cong\left(C, \partial_{\epsilon^{\prime}}\right)$.

Proof We divide the crossings of $L$ into two equivalence classes as follows. Fix one of the two checkerboard colorings of the diagram. We can then define a function $\theta$ from the crossings into \pm 1 depending on how the crossing is colored, as illustrated in Figure 6.

Fix some initial orientation $\mathfrak{o}$ for the crossings in a knot projection, and a sign assignment $\epsilon$. Consider next a different set of orientations on the initial crossings, specified by $\theta \cdot \mathfrak{o}$ (ie if $c$ is some crossing with type $\theta(c)=+1$, then $\mathfrak{o}^{\prime}(c)$ is the same as $\mathfrak{o}(c)$, whereas if $\theta(c)=-1$, then the two orientations point in opposite directions). Clearly, this change of orientations swaps squares of type $X$ (for one orientation) with 

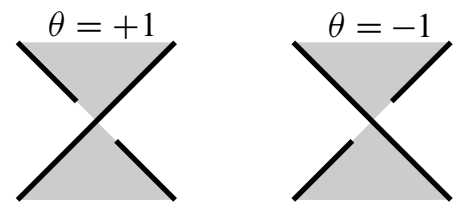

Figure 6: Black and white coloring. Given a checkerboard coloring of the projection, we can introduce a function $\theta: \mathcal{X} \rightarrow\{ \pm 1\}$ as illustrated.

those of type $Y$ (for the other). Moreover, it preserves the types of all other squares. (Note that there are only two squares not of type $X$ or $Y$ whose types depend on the orientations of the two arcs; for those two squares, the arcs represent crossings in the same equivalence class.) Thus, if we view $\epsilon$ as a type $X$ sign assignment for orientation $\mathfrak{o}$, then $\epsilon$ can also be viewed as a type $Y$ assignment for the orientation $\mathfrak{o}^{\prime}$. The lemma now follows from Lemma 2.3.

\section{Topological invariance}

In this section, we check that $\mathrm{Kh}^{\prime}(L)$ is invariant under the three Reidemeister moves, thus verifying Theorem 1.4. The argument is more or less the same one used by Khovanov to prove invariance of the ordinary $s l(2)$ homology [4]; see also [6] and [7]. We follow Bar-Natan's exposition in [1].

Specifically, we must verify independence of odd Khovanov homology under the three Reidemeister moves. To this end, in view of Lemmas 2.2, 2.3 and 2.4, we are free to choose the crossing orientations and edge assignments as we please.

Proposition 3.1 The homology groups of $C(\mathcal{D})$ remain invariant as the diagram undergoes a Reidemeister move of type I.

Proof Suppose that $\mathcal{D}^{\prime}$ is obtained from $\mathcal{D}$ by a Reidemeister move of type I, as shown in Figure 7. For concreteness, we focus on the Reidemeister move illustrated in the top row.

We can split the $\mathbb{Z}$-module $C\left(\mathcal{D}^{\prime}\right) \cong C\left(\mathcal{D}_{0}\right) \oplus C\left(\mathcal{D}_{1}\right)$, where $\mathcal{D}_{0}$ denotes the disjoint union of $\mathcal{D}$ with an unknotted component $O$, and $\mathcal{D}_{1}$ is identified with the original diagram $\mathcal{D}$. Indeed, if $\epsilon$ is an edge assignment for $\mathcal{D}$, we can use it to induce an edge assignment $\epsilon^{\prime}$ on $\mathcal{D}^{\prime}$ by declaring its restriction to $\mathcal{D}_{1}$ to be identified with $-\epsilon$; its restriction to $\mathcal{D}_{0}$ to agree with $\epsilon$, and its assignment to the edges connecting $\mathcal{D}_{0}$ to $\mathcal{D}_{1}$ to be all +1 . The map $\epsilon^{\prime}$ is an edge assignment of the same type as $\epsilon$, since all the squares involving the edges connecting $\mathcal{D}_{0}$ and $\mathcal{D}_{1}$ are of type $C$ (there is exactly one edge leaving the distinguished unknotted component). 

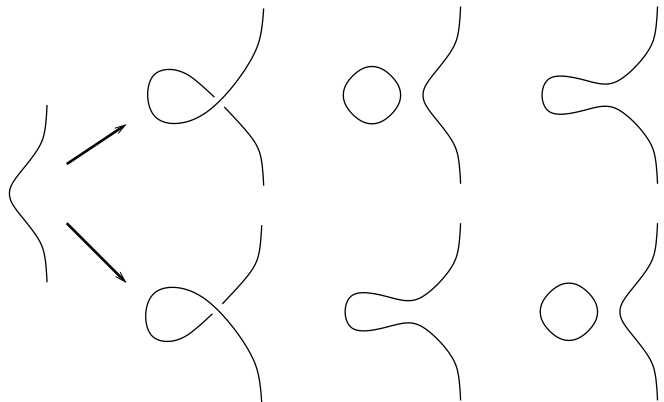

Figure 7: Starting with the initial picture on the left, we can perform two types of Reidemeister I move, as illustrated in the next column. After that, we display the 0 - and 1 -resolutions respectively.

In effect, we have identified $C\left(\mathcal{D}^{\prime}\right)$ with the mapping cone of a map

$$
D: C\left(\mathcal{D}_{0}\right) \longrightarrow C\left(\mathcal{D}_{1}\right),
$$

where

$$
\left(C\left(\mathcal{D}_{0}\right), \partial_{0}\right) \cong(C(\mathcal{D}), \partial) \otimes \Lambda^{*}\left(\mathbb{Z} \cdot v_{0}\right) \quad \text { and } \quad\left(C\left(\mathcal{D}_{1}\right), \partial_{1}\right) \cong(C(\mathcal{D}),-\partial),
$$

and the map $D$ identifies $v_{0}$ with $v_{1}$ (where $v_{0}$ denotes the unknotted component in $\mathcal{D}_{0}$ and $v_{1}$ denotes the component which is connected to it). This chain complex is clearly quasi-isomorphic to the subcomplex $\left(v_{1}-v_{0}\right) \wedge\left(C\left(\mathcal{D}_{0}\right), \partial_{0}\right)$, which in turn is identified with the complex $C(\mathcal{D})$. This establishes the stated isomorphism for one of the two types of Reidemeister move.

Proposition 3.2 The homology groups of $C(\mathcal{D})$ remain invariant as the diagram undergoes a Reidemeister move of type II.

Proof We consider the diagram for the second Reidemeister move, following notation suggested in Figure 8. The resolutions after the Reidemeister move have four types, $\mathcal{D}_{i, j}$ with $i, j \in\{0,1\}$, so that $\mathcal{D}_{00}$ and $\mathcal{D}_{11}$ are identified, $\mathcal{D}_{10}$ is obtained from $\mathcal{D}_{00}$ by inserting an unknotted component, and $\mathcal{D}_{01}$ is identified with the diagram before the Reidemeister move. The chain complex after the Reidemeister move can be written in the following form:

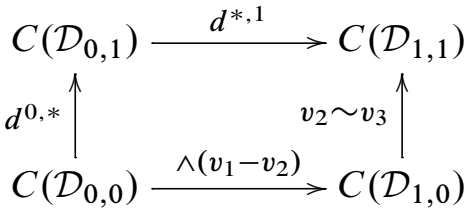


(We have labeled $v_{1}$ to be the component belonging to the top of the Reidemeister II move, $v_{2}$ to be the middle component, and $v_{3}$ to be the bottom.) In defining this complex, we have implicitly picked an edge assignment $\epsilon^{\prime}$ for $\mathcal{D}^{\prime}$ (the diagram after the second Reidemeister move.) It is easy to see that the restriction of $\epsilon^{\prime}$ to $C\left(\mathcal{D}_{1,0}\right)$ will be an edge assignment for $\mathcal{D}$.

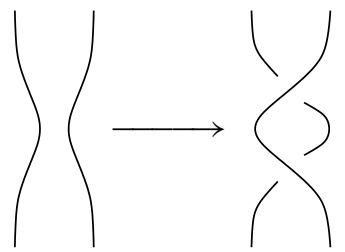

01
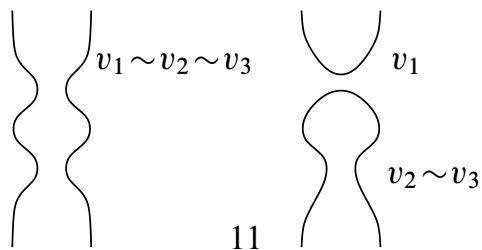

00
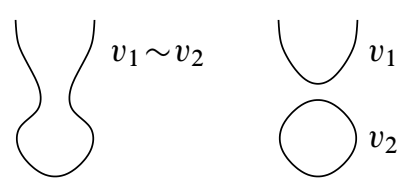

10

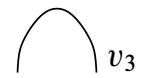

Figure 8: Starting with the initial picture on the left, we can perform a Reidemeister move to obtain the second picture. We have then illustrated (and labeled) the four resolutions of this diagram.

Let $X \subset C\left(\mathcal{D}_{0,1}\right)$ be the kernel of the contraction map with $v_{2}^{*}$. (In other words, $X$ is generated by those exterior products which do not contain $v_{2}$ as a factor.) It is not difficult to see that $A=X \oplus C\left(\mathcal{D}_{1,1}\right)$ is an acyclic subcomplex of $C\left(\mathcal{D}^{\prime}\right)$. The quotient complex $C\left(\mathcal{D}^{\prime}\right) / A$ will have the same homology as $C\left(\mathcal{D}^{\prime}\right)$. This quotient is of the form

$$
\begin{aligned}
& C\left(\mathcal{D}_{0,1}\right) \\
& d^{0, *} \uparrow^{\uparrow}\left(\mathcal{D}_{0,0}\right) \stackrel{\wedge\left(v_{1}-v_{2}\right)}{\longrightarrow} C\left(\mathcal{D}_{1,0}\right) / X .
\end{aligned}
$$

This complex clearly has $C\left(\mathcal{D}_{0,1}\right)$ as a subcomplex, and it is easy to check that the quotient $\left(C\left(\mathcal{D}^{\prime}\right) / A\right) / C\left(\mathcal{D}_{0,1}\right)$ is acyclic. Thus $C\left(\mathcal{D}_{0,1}\right)$ will have the same homology as $C\left(\mathcal{D}^{\prime}\right)$.

Proposition 3.3 The homology groups of $C(\mathcal{D})$ remain invariant as the diagram undergoes a Reidemeister move of type III. 
Proof To see how, we consider the cube of resolutions before the Reidemeister move of type III is performed. This is illustrated in Figure 9. After contracting the indicated arrows, we obtain an intermediate chain complex illustrated in Figure 10. This in turn is related to the cube of resolutions for the projection after the Reidemeister move is performed, by contracting two edges as indicated in Figure 12.

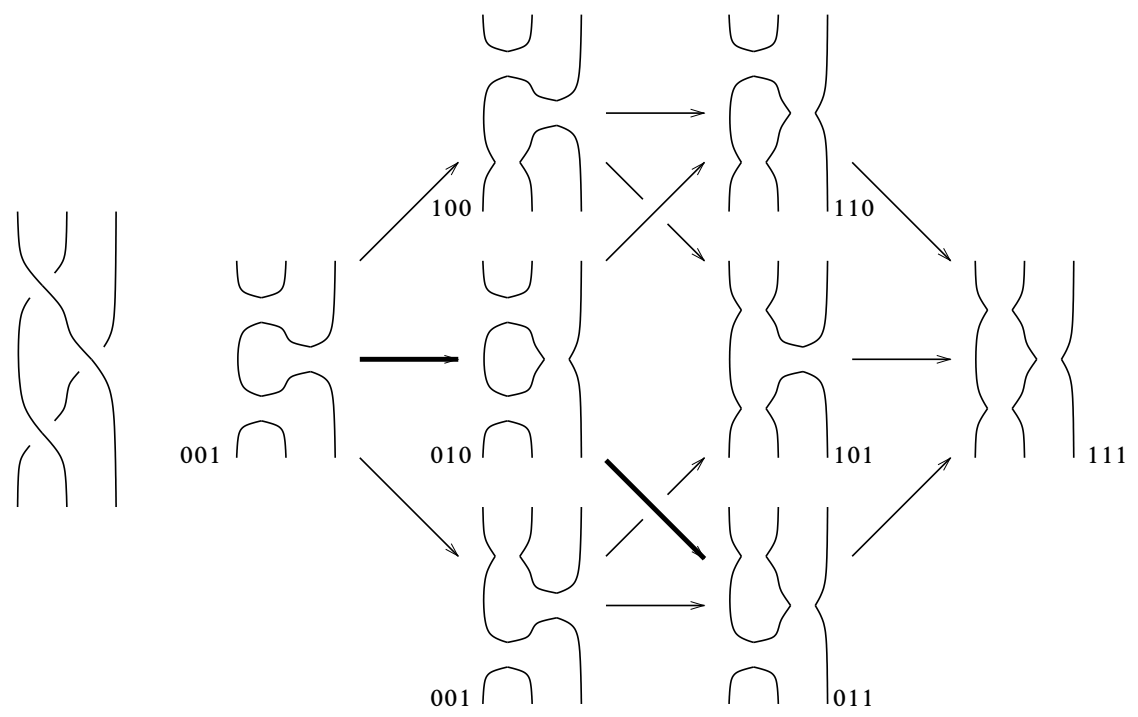

Figure 9: Consider the local picture on the left. We obtain a corresponding cube of resolutions which is pictured on the right, where the resolutions are labeled by their corresponding resolution vector. The thick arrow is contracted to obtain the chain complex from Figure 10 below.

We give a more precise version of this argument, with signs. Consider the diagram illustrated in Figure 9. This complex has a quotient, consisting of a mapping cone of a map

$$
d: C(000) \longrightarrow C(010) \wedge \mu
$$

where here $\mu$ denotes the unknotted component in the resolution indicated by the vector 010. Clearly, this map $d$ is an isomorphism, and hence the full complex from Figure 9 is isomorphic to the subcomplex illustrated on the left in Figure 11.

Observe that $C(110) \cong C(011)$. Fix an edge assignment $\epsilon_{110}=\epsilon_{011}$ on these two cubes, and extend it to an edge assignment $\epsilon$ on the entire cube of resolutions. (This is possible, since the quotient space in which we collapse $C(110)$ and $C(011)$ to a point has vanishing $H^{2}$.) The five term complex $P$ shown in Figure 10 is obtained by multiplying the edge maps by the indicated signs. For example, the differential from $A$ 


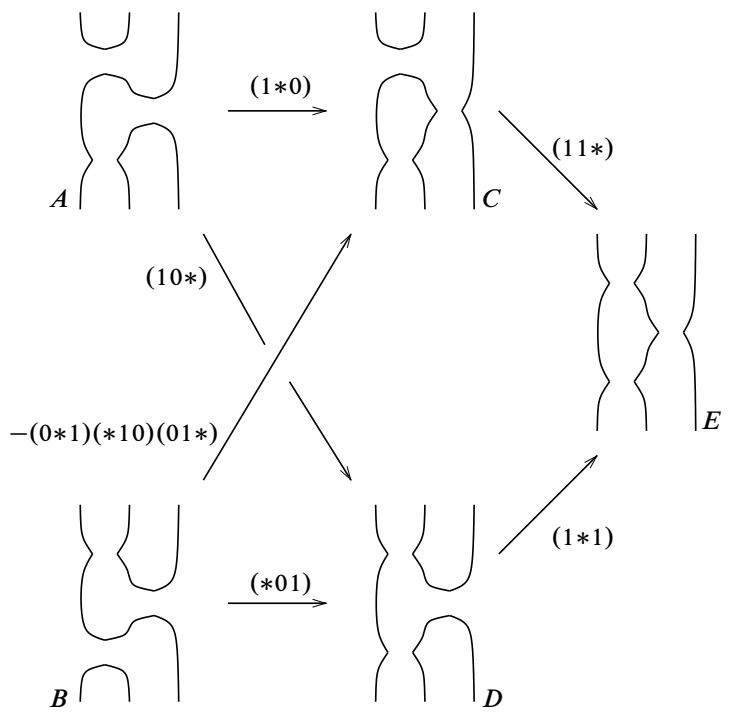

Figure 10: After contraction

to $D$ is multiplied by the sign $\epsilon(10 *)$ from the hypercube of Figure 9; similarly, the differential from $B$ to $C$ is multiplied by the sign $-\epsilon(0 * 1) \epsilon(01 *) \epsilon(* 10)$.

Consider the map $\Phi$ from the complex on the left in Figure 11 to the complex in Figure 10 defined as follows. $\Phi$ induces the natural identification from $C(100)$ to $A$; $\Phi$ induces the natural identification from $C(001)$ to $B ; \Phi$ induces the identification of $C(110)$ to $C ; \Phi$ induces the identification of $C(011)$ with $C$ times $-\epsilon(* 10) \epsilon(01 *)$; $\Phi$ induces the identification of $C(101)$ with $D ; \Phi$ induces the identification of $C(111)$ with $E$. Finally, the restriction of $\Phi$ to $C(010)$ is trivial. It is straightforward to verify that $\Phi$ is a chain map.

We now define a chain map $\Psi$ from the right complex $R$ to the left complex $L$. Note that the right complex consists of two terms, one of which is identified with $C(010) / \mu$, and the second of which is naturally identified with $C(110)$ or $C(011)$. The differential is an isomorphism (and hence the right complex is acyclic).

Restricted to $C(010) / \mu$, the map $\Psi$ is an isomorphism onto the corresponding term in the cube of resolution; its restriction to the second term is identified with the diagonal identification onto $C(110)$ and $C(011)$; in fact, we multiply the component in $C(110)$ with the sign of the edge $\epsilon(1 * 0)$ and the component in $C(011)$ with the sign of the edge $\epsilon(0 * 1)$. It is straightforward to verify that $\Psi$ is a chain map. Moreover, it is straightforward to verify the short exact sequence

$$
0 \longrightarrow R \stackrel{\Psi}{\longrightarrow} L \stackrel{\Phi}{\longrightarrow} P \longrightarrow 0 .
$$


This completes the proof that the homology of the complex before the Reidemeister move is identified with the complex pictured in Figure 10.
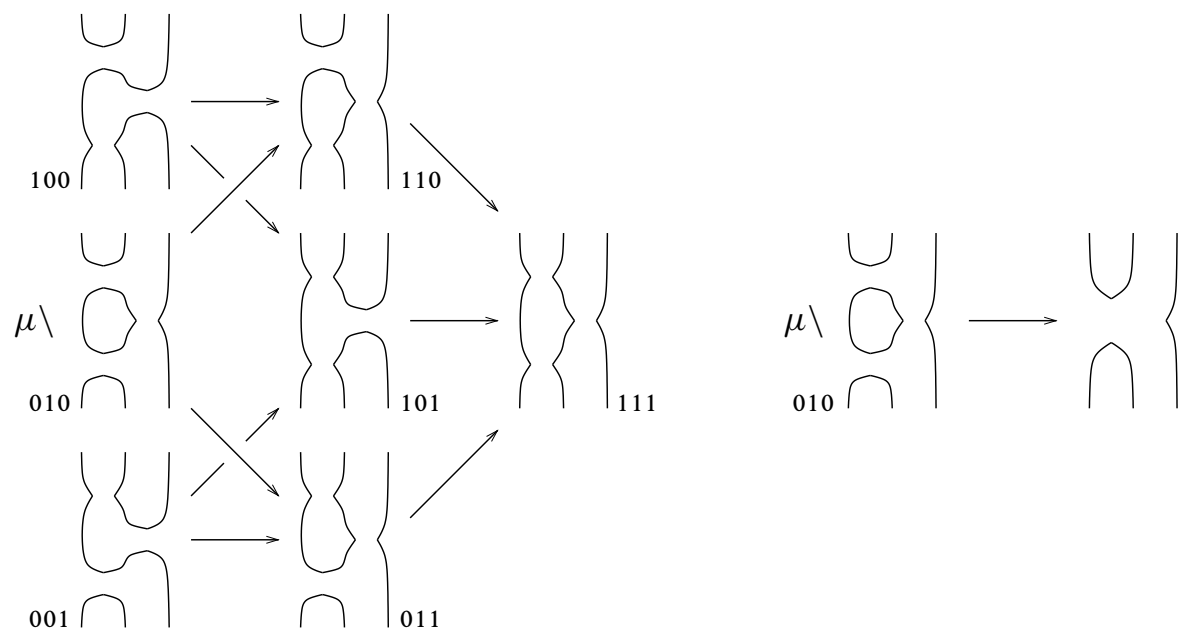

Figure 11: Intermediate states. On the left is the quotient complex after the rightmost thick arrow from Figure 9 is contracted. There is a natural map to the mapping cone illustrated on the right.

We now give a more intrinsic description of the signs in the chain complex of Figure 10. To this end, consider the simplicial complex $\mathcal{P}$ obtained as the product of the twodimensional simplicial complex appearing in Figure 10 (5 vertices, 6 edges and 2 faces) with a cube of the appropriate dimension. It is easy to see that $\mathcal{P}$ is contractible. Let $\psi$ be the two-dimensional cochain on $\mathcal{P}$ with $\mathbb{Z} / 2 \mathbb{Z}$-coefficients which takes nontrivial values on faces of type $C$ or $Y$ (as in the proof of Lemma 1.3.) Above, we have described an explicit edge assignment $\epsilon$ on the edges of $\mathcal{P}$ for which $d \epsilon=\psi$. As in Lemma $2.2, H^{1}(\mathcal{P}, \mathbb{Z} / 2 \mathbb{Z})=0$, so if $\epsilon_{1}$ and $\epsilon_{2}$ are any two one-dimensional cochains with $\delta \epsilon_{1}=\delta \epsilon_{2}=\psi$, then they determine isomorphic chain complexes.

Now consider the diagram we obtain after making a Reidemeister III move. This diagram and the associated chain complex are shown in Figure 12. We can contract the boldface edges (just as we did with the complex in Figure 9) to obtain a new chain complex which agrees (up to sign) with $P$. To show that the two complexes are genuinely isomorphic, it is enough to check that the corresponding obstruction cocycles $\psi, \psi^{\prime} \in C^{2}(\mathcal{P}, \mathbb{Z} / 2)$ are the same. If we choose the upward pointing orientation at all of the crossings in Figures 9 and 12 (and use the same orientations at crossings not shown in the diagram), then this is easily seen to be the case. 


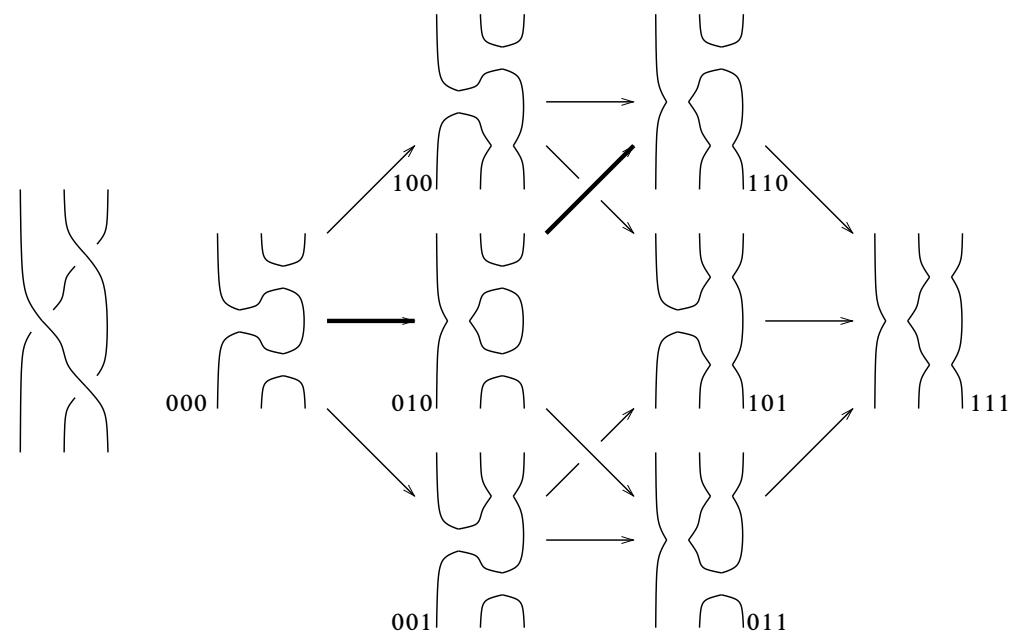

Figure 12: This is the complex after Reidemeister III is performed on Figure 9. Contracting the thick arrows, we obtain a five term complex which is isomorphic to the one from Figure 10.

Proof of Theorem 1.4 According to a classical result of Reidemeister, an invariant of link projections which is unchanged under the three Reidemeister moves is in fact a link invariant; see for example Burde and Zieschang [3]. (Note that it is actually sufficient to check invariance under the three moves considered here, since the "other" Reidemeister I and III moves can be obtained by composing the moves we have studied with some Reidemeister II moves.) Thus, $\mathrm{Kh}^{\prime}$ is a link invariant according to Propositions 3.1, 3.2 and 3.3.

\section{Basic properties}

In this section, we sketch the proofs of the properties stated in the introduction. We begin by giving two equivalent definitions of the reduced homology.

Let $\Lambda_{\circ}^{*} V(\mathcal{D})$ be the subalgebra of $\Lambda^{*} V(\mathcal{D})$ generated by the kernel of the map $V(\mathcal{D}) \rightarrow \mathbb{Z}$ defined by

$$
\sum n_{i} a_{i} \longmapsto \sum n_{i}
$$

There is a corresponding subcomplex $\bar{C}(\mathcal{D}) \subset C(\mathcal{D})$.

Given a generic point $p$ on the knot projection, we also have a subalgebra

$$
a_{p} \wedge \Lambda^{*} V(\mathcal{D}(I)) \subset \Lambda^{*} V(\mathcal{D}(I)),
$$


where $a_{p}$ denotes the component of $\mathcal{D}(I)$ containing $p$. There is a corresponding subcomplex

$$
\bar{C}^{(p)}(\mathcal{D})=a_{p} \wedge C(\mathcal{D}) \subset C(\mathcal{D})
$$

Lemma 4.1 Wedge product with $a_{p}$ induces an isomorphism of $\Lambda_{\circ}^{*} V(\mathcal{D})$ with $a_{p} \wedge \Lambda^{*} V(\mathcal{D})$. In fact, this induces an isomorphism of complexes $\bar{C}^{(p)}(\mathcal{D}) \cong \bar{C}(\mathcal{D})$.

Proof Straightforward.

Proof of Proposition 1.8 If $\mathcal{D}$ is a diagram of the link $L$, we define $\overline{\mathrm{Kh}}^{\prime}(L)$ to be $H_{*}(\bar{C}(\mathcal{D}))$. Consider the disjoint union of $\mathcal{D}$ with an unknotted link $O$. Taking $p$ to lie on the unknotted component, it is easy to see that $\bar{C}^{(p)}(\mathcal{D} \cup O) \cong C(\mathcal{D})$. On the other hand, taking $p$ elsewhere, we see that $\bar{C}^{(p)}(\mathcal{D} \cup O) \cong \bar{C}^{(p)}(\mathcal{D}) \oplus \bar{C}^{(p)}(\mathcal{D})$. Since $\bar{C}^{(p)}(\mathcal{D}) \cong \bar{C}(\mathcal{D})$, and the latter chain complex is independent of the placement of the basepoint, we conclude that $C(\mathcal{D}) \cong \bar{C}(\mathcal{D}) \oplus \bar{C}(\mathcal{D})$. Passing to homology, we obtain the stated result.

Proof of Proposition 1.7 The mod two reduction of $\Lambda^{*} V(\mathcal{D})$, equipped with our multiplication and comultiplication maps (whose mod two reduction no longer depends on the choice of orientations at the crossings) coincides with the mod two reduction of Khovanov's TQFT. It follows at once that if $\operatorname{CKh}(\mathcal{D})$ denotes Khovanov's complex, then $\operatorname{CKh}(\mathcal{D}) \otimes \mathbb{Z} / 2 \mathbb{Z} \cong C(\mathcal{D}) \otimes \mathbb{Z} / 2 \mathbb{Z}$. The proposition now follows from the universal coefficient theorem.

Proof of Proposition 1.5 This a direct consequence of Proposition 1.7 and the analogous formula for the ordinary Khovanov homology.

Proof of Proposition 1.6 By construction, $\mathrm{Kh}^{\prime}(L)$ is the homology of a mapping cone of $C\left(L_{0}\right)$ to $C\left(L_{1}\right)$. The stated exact sequence is an application of the long exact sequence of a mapping cone, with appropriate shifts in gradings. A corresponding result for $\overline{\mathrm{Kh}}^{\prime}$ is also apparent, using the definition of the reduced complex $\bar{C}$.

\section{Calculations}

In this section, we give a few computations of the odd Khovanov homology. In light of Proposition 1.8, we may as well restrict our attention to the reduced groups $\overline{\mathrm{Kh}}^{\prime}(L)$. For the simplest knots and links, these groups exhibit a pattern which is familiar from the usual Khovanov homology: 
Definition 5.1 $\overline{\mathrm{Kh}}^{\prime}(L)$ is said to be $\sigma$-thin if ${\overline{\mathrm{Kh}^{\prime}}}^{\prime}(L)_{m, s}=0$ when $s-2 m \neq \sigma(L)$.

Here, our sign convention for the signature is that positive links have positive signature. In analogy with Lee's Theorem [9] on the ordinary cohomology of alternating knots, we have:

Proposition 5.2 If $L$ admits a connected alternating projection, then $\overline{\mathrm{Kh}}^{\prime}(L)$ is $\sigma$-thin.

Proof The standard proof of this result for the ordinary Khovanov homology relies on two facts: first, that the reduced homology $\overline{\mathrm{Kh}}$ satisfies a skein exact sequence like the one in Proposition 1.6, and second, that $\overline{\mathrm{Kh}}$ of the unknot is supported in bigrading $(0,0)$. Since both of these hold for $\overline{\mathrm{Kh}}^{\prime}$ as well, the proof goes through without change.

More generally, the same result holds if $L$ is quasialternating in the sense of [12] (cf [10]).

In light of Proposition 1.5, it is not difficult to see that if $\overline{\mathrm{Kh}}^{\prime}(L)$ is $\sigma$-thin, it is completely determined by the Jones polynomial and signature of $L$. Since the same result is true for $\overline{\mathrm{Kh}}$, we see that $\overline{\mathrm{Kh}}^{\prime}(L) \cong \overline{\mathrm{Kh}}(L)$ whenever $L$ is alternating. The analogous statement for the unreduced homology is emphatically not true; $\overline{\mathrm{Kh}}$ and $\mathrm{Kh}$ are related by a long exact sequence analogous to Proposition 1.8 ,

$$
\longrightarrow \overline{\mathrm{Kh}}_{m, s+1}(L) \longrightarrow \mathrm{Kh}_{m, s}(L) \longrightarrow \overline{\mathrm{Kh}}_{m, s-1}(L) \stackrel{\partial}{\longrightarrow} \overline{\mathrm{Kh}}_{m+1, s+1}(L) \longrightarrow,
$$

but the boundary map in this sequence is almost never 0 . The difference between the two is already evident with the trefoil knot, for which $\mathrm{Kh}^{\prime}$ has rank 6 and $\mathrm{Kh}$ has rank 4.

To find examples where $\overline{\mathrm{Kh}}^{\prime}(L) \neq \overline{\mathrm{Kh}}(L)$, we resort to computer calculations. Using a Mathematica program based on Bar-Natan's original program for computing the Khovanov homology [1], we computed $\overline{\mathrm{Kh}}^{\prime}(K) \otimes \mathbb{Q}$ for all nonalternating knots $K$ with fewer than 12 crossings. The first knot which is not quasialternating (and thus the first for which we might expect the two to differ) is the $(3,4)$ torus knot, number $8_{19}$ in the Rolfsen table. Indeed, we find that $\overline{\mathrm{Kh}}^{\prime}\left(8_{19}\right)$ has rank 3 , with graded Poincaré polynomial

$$
\overline{K h}^{\prime}\left(8_{19}\right)(q, t)=\sum_{m, s} t^{m} q^{s} \operatorname{dim} \overline{\mathrm{Kh}}_{m, s}^{\prime}\left(8_{19}\right) \otimes \mathbb{Q}=q^{6}+q^{10} t^{2}+q^{16} t^{5},
$$

while $\overline{\mathrm{Kh}}\left(8_{19}\right)$ is known to have rank 5 [15], with graded Poincaré polynomial $\overline{K h}\left(8_{19}\right)(q, t)=\sum_{m, s} t^{m} q^{s} \operatorname{dim} \overline{\mathrm{Kh}}_{m, s}\left(8_{19}\right) \otimes \mathbb{Q}=q^{6}+q^{10} t^{2}+q^{12} t^{3}+q^{12} t^{4}+q^{16} t^{5}$. 
This computation is somewhat disappointing, since it indicates that $\mathrm{Kh}^{\prime}$ cannot possess a canceling differential analogous to the Lee differential [9] and its generalizations [18].

The other nonquasialternating knot with fewer than 10 crossings has Rolfsen number $9_{42} \cdot \overline{\mathrm{Kh}}^{\prime}\left(9_{42}\right) \otimes \mathbb{Q}$ turns out to be $\sigma$-thin. However, the corresponding statement over $\mathbb{Z}$ cannot be true, since if we use $\mathbb{Z} / 2$-coefficients, $\overline{\mathrm{Kh}^{\prime}}\left(9_{42}\right)$ reduces to $\overline{\mathrm{Kh}}\left(9_{42}\right)$, which is not $\sigma$-thin. These two examples exhibit a general trend which continues with the 10 and 11-crossing knots; namely, that the rank of $\overline{\mathrm{Kh}}^{\prime}(K)$ tends to be smaller than that of $\overline{\mathrm{Kh}}(K)$. The table below compares the dimensions of $\overline{\mathrm{Kh}}^{\prime} \otimes \mathbb{Q}$ and $\overline{\mathrm{Kh}} \otimes \mathbb{Q}$ for the nonalternating 10-crossing knots, omitting those knots for which both groups are $\sigma$-thin:

\begin{tabular}{ccc|ccc|ccc}
$K$ & $\overline{\mathrm{Kh}}^{\prime}(K)$ & $\overline{\mathrm{Kh}}(K)$ & $K$ & $\overline{\mathrm{Kh}^{\prime}}(K)$ & $\overline{\mathrm{Kh}}(K)$ & $K$ & $\overline{\mathrm{Kh}^{\prime}}(K)$ & $\overline{\mathrm{Kh}}(K)$ \\
\hline $10_{124}$ & 3 & 7 & $10_{139}$ & 7 & 11 & $10_{153}$ & 9 & 17 \\
$10_{128}$ & 11 & 13 & $10_{145}$ & 7 & 13 & $10_{154}$ & 17 & 21 \\
$10_{132}$ & 5 & 11 & $10_{152}$ & 15 & 19 & $10_{161}$ & 9 & 13 \\
$10_{136}$ & 15 & 17 & & & & & &
\end{tabular}

Among the $10-$ crossing knots, $\overline{\mathrm{Kh}}^{\prime}$ is $\sigma$-thin whenever $\overline{\mathrm{Kh}}$ is $\sigma$-thin; when $\overline{\mathrm{Kh}}$ is not $\sigma$-thin, the rank of $\overline{\mathrm{Kh}}^{\prime}$ is strictly smaller. The same pattern also holds for the 11-crossing knots. Below, we tabulate the Poincaré polynomials of those 10-crossing knots for which $\overline{\mathrm{Kh}}^{\prime}$ is not $\sigma$-thin:

$\overline{K h}^{\prime}\left(10_{124}\right)=q^{8}+q^{12} t^{2}+q^{20} t^{7}$,

$\overline{K h}^{\prime}\left(10_{139}\right)=q^{8}+q^{12} t^{2}+q^{16} t^{5}+q^{18} t^{6}+q^{20} t^{7}+q^{22} t^{8}+q^{24} t^{9}$,

$\overline{K h}^{\prime}\left(10_{145}\right)=q^{-20} t^{-9}+q^{-18} t^{-8}+q^{-16} t^{-7}+q^{-14} t^{-6}+q^{-8} t^{-3}+q^{-8} t^{-2}+q^{-4}$,

$\overline{K h}^{\prime}\left(10_{152}\right)=q^{-26} t^{-10}+2 q^{-24} t^{-9}+2 q^{-22} t^{-8}+3 q^{-20} t^{-7}$

$$
+2 q^{-18} t^{-6}+2 q^{-16} t^{-5}+q^{-14} t^{-4}+q^{-12} t^{-2}+q^{-8}
$$

$\overline{K h^{\prime}}\left(10_{153}\right)=q^{-10} t^{-5}+q^{-8} t^{-4}+q^{-6} t^{-3}+q^{-4} t^{-2}+1+q^{2} t^{2}+q^{4} t^{3}+q^{6} t^{4}+q^{8} t^{5}$, $\overline{K h}^{\prime}\left(10_{154}\right)=q^{6}+q^{10} t^{2}+q^{10} t^{3}+2 q^{12} t^{4}+2 q^{14} t^{5}$

$$
+2 q^{16} t^{6}+3 q^{18} t^{7}+2 q^{20} t^{8}+2 q^{22} t^{9}+q^{24} t^{10},
$$

$\overline{K h}^{\prime}\left(10_{161}\right)=q^{-22} t^{-9}+q^{-20} t^{-8}+q^{-18} t^{-7}+q^{-16} t^{-6}$

$$
+q^{-14} t^{-5}+q^{-12} t^{-4}+q^{-10} t^{-3}+q^{-10} t^{-2}+q^{-6} \text {. }
$$




\section{References}

[1] D Bar-Natan, On Khovanov's categorification of the Jones polynomial, Algebr. Geom. Topol. 2 (2002) 337-370 MR1917056

[2] J M Bloom, Odd Khovanov homology is mutation invariant, Math. Res. Lett. 17 (2010) 1-10 MR2592723

[3] G Burde, H Zieschang, Knots, 2nd edition, de Gruyter Studies in Math. 5, Walter de Gruyter \& Co., Berlin (2003) MR1959408

[4] M Khovanov, A categorification of the Jones polynomial, Duke Math. J. 101 (2000) 359-426 MR1740682

[5] M Khovanov, Patterns in knot cohomology. I, Experiment. Math. 12 (2003) 365-374 MR2034399

[6] M Khovanov, L Rozansky, Matrix factorizations and link homology, Fund. Math. 199 (2008) 1-91 MR2391017

[7] M Khovanov, L Rozansky, Matrix factorizations and link homology. II, Geom. Topol. 12 (2008) 1387-1425 MR2421131

[8] P Kronheimer, T Mrowka, Monopoles and three-manifolds, New Mathematical Monographs 10, Cambridge Univ. Press (2007) MR2388043

[9] E S Lee, An endomorphism of the Khovanov invariant, Adv. Math. 197 (2005) 554-586 MR2173845

[10] C Manolescu, P Ozsváth, On the Khovanov and knot Floer homologies of quasialternating links, from: "Proc. Gökova Geometry-Topology Conf. 2007”, (S Akbulut, T Önder, R J Stern, editors), GGT, Gökova (2008) 60-81 MR2509750

[11] V O Manturov, Khovanov homology for virtual knots with arbitrary coefficients, J. Knot Theory Ramifications 16 (2007) 345-377 MR2320160

[12] P Ozsváth, Z Szabó, On the Heegaard Floer homology of branched double-covers, Adv. Math. 194 (2005) 1-33 MR2141852

[13] K Putyra, Cobordisms with chronologies and a generalisation of the Khovanov complex arXiv: 1004.0889

[14] J Rasmussen, Knot polynomials and knot homologies, from: "Geometry and topology of manifolds", (H U Boden, I Hambleton, A J Nicas, B D Park, editors), Fields Inst. Commun. 47, Amer. Math. Soc. (2005) 261-280 MR2189938

[15] A N Shumakovitch, KhoHo, computer program (2003) Available at https:// github.com/AShumakovitch/KhoHo

[16] A N Shumakovitch, Patterns in odd Khovanov homology, J. Knot Theory Ramifications 20 (2011) 203-222 MR2777025

[17] Z Szabó, A geometric spectral sequence on odd Khovanov homology, preprint 
[18] P R Turner, Calculating Bar-Natan's characteristic two Khovanov homology, J. Knot Theory Ramifications 15 (2006) 1335-1356 MR2286127

Department of Mathematics, Massachusetts Institute of Technology

Cambridge, MA 02139, USA

Department of Pure Mathematics and Mathematical Statistics

University of Cambridge, Cambridge CB3 OWB, UK

Department of Mathematics, Princeton University

Fine Hall, Washington Road, Princeton, NJ 08544, USA

petero@math.princeton.edu, J.Rasmussen@dpmms.cam.ac.uk, szabo@math.princeton.edu

Received: 9 September 2008 Revised: 11 July 2012 